\title{
The Sialome-Far More Than the Sum of Its Parts
}

\author{
Miriam Cohen and Ajit Varki
}

\begin{abstract}
The glycome is defined as the glycan repertoire of cells, tissues, and organisms, as found under specified conditions. The vastly diverse glycome is generated by a nontemplate driven biosynthesis, which is indirectly encoded in the genome, and very dynamic. Due to this overwhelming diversity, glycomic analysis must be approached at different hierarchical levels of complexity. In this review five such levels of complexity and the experimental approaches used for analysis at each level are discussed for a subclass of the glycome: the sialome. The sialome, in analogy to the canopy of a forest, covers the cell membrane with diverse array of complex sialylated structures. Sialome complexity includes modification of sialic acid core structure (the leaves and flowers), the linkage to the underlying sugar (the stems), the identity, and arrangement of the underlying glycans (the branches), the structural attributes of the underlying glycans (the trees), and finally, the spatial organization of the sialoglycans in relation to components of the intact cell surface (the forest). Understanding the full complexity of the sialome thus requires combined analyses at multiple levels, that is, the sialome is far more than the sum of its parts.
\end{abstract}

\section{Introduction}

G LyCOCONJUGATES ARE FOUND in abundance on all cell surfaces in nature, forming a dense array of glycans, originally known as the glycocalyx. Localized at the outer face of the cell, many glycoconjugates mediate cell recognition and facilitate initial cellular interactions in the context of numerous physiological and pathological processes. Profiling of the diverse glycan repertoires of cells, tissues and organisms under specific conditions is typically termed "glycomics." Compared with genomics and proteomics, glycomic studies are faced with many additional challenges. This difficulty is attributed to a large variety of monosaccharide units, different types of linkages, and branching structures. In contrast to nucleotides and proteins which are linear polymers, each characteristically contain one basic type of linkage between monomers, each monosaccharide can theoretically generate $\alpha$ or $\beta$ linkages to any one of several positions on another monosaccharide. Thus, three different nucleotides or amino acids can only generate six trimers, while three different hexoses can form at least 1,056 and up to 27,648 unique trisaccharides. Fortunately, naturally occurring biological macromolecules are so far known to contain relatively few of the possible monosaccharide units, in a limited number of combinations (Varki and Sharon, 2009). In addition, glycan biosynthesis is not template driven and only encoded indirectly in the genome. Furthermore, a range of variation in glycan structures can be found on any given gly- cosylation site on a given glycoprotein (glycosylation microheterogeneity) (Kornfeld and Kornfeld, 1985). Thus a given protein encoded by a single gene can have multiple "glycoforms" (Rademacher et al., 1988). Due to this overwhelming diversity, glycomic analysis must be approached at different hierarchical levels of complexity, and advanced profiling and structural characterization strategies are constantly being developed for this task.

This review will focus on a subclass of the glycome: the sialome. The sialome is defined as the "total complement of sialic acid types and linkages and their mode of presentation on a particular organelle, cell, tissue, organ or organism-as found at a particular time and under specific conditions" (Varki and Angata, 2006). Sialic acids (Sias) are a family of nine-carbon backbone sugars that are typically found attached to the outermost ends of glycoconjugate chains (Angata and Varki, 2002; Kelm and Schauer, 1997; Schauer, 1982, 2009; Troy, 1992; Varki, 1992, 2007, 2008; Varki and Schauer, 2009), and provide an extreme example of glycan diversity, both in structure and function. The sialome can be analyzed on multiple hierarchical levels of complexity: modification of Sia core structure, the linkage of Sia to the underlying sugar, the identity and arrangement of the underlying sugars, structural attributes of the underlying sugar, spatial organization of the Sia, and higher level of cellular organization (Lewis and Varki, 2010). This review will discuss the sialome and the experimental approaches used for 
analysis at each level of its complexity. An analogy can be made to the study of a forest canopy, which also needs to be examined at different levels of complexity, each of which provides valuable information, all necessary for complete understanding of the forest.

\section{Complexity Level 1: Sialic Acid Structure (the Leaves and Flowers)}

The two major Sia core structures are 2-keto-3deoxynononic-acid (Kdn; Fig. 1a) and neuraminic acid (Neu, Fig. 1b) (Angata and Varki, 2002; Varki and Schauer, 2009), sharing nine carbons and differing at the C5-position. Two derivatives of Neu are the most common Sia structures found on mammalian cells: $N$-acetylneuraminic acid (Neu5Ac, Fig. 1c) and its hydroxylated form, $N$-glycolylneuraminic acid (Neu5Gc;Fig. 1d) (Angata and Varki, 2002). Among mammals, humans are a known exception in their lack of Neu5Gc, due to an inactivation mutation in a hydroxylase, which modifies CMP-Neu5Ac to CMP-Neu5Gc (Varki, 2007). Most of the other Sias arise from $\mathrm{O}$-substitution of one or more of the hydroxyl groups of Kdn, Neu, Neu5Ac, and Neu5Gc. Natural modification of Sia core structures yield over 50 variations, which include esterification (with acetyl, lactyl, sulfate or phosphate), $\mathrm{O}$-methylation, lactonization, or lactamization (Angata and Varki, 2002; Kelm and Schauer, 1997; Schauer, 1982, 2009; Troy, 1992; Varki, 1992; Varki and Schauer, 2009). These modifications can dramatically affect Sia recognition by glycan binding proteins (GBPs), for example, human and mouse sialoadhesin (Siglec-1) strongly prefers to bind Neu5Ac over Neu5Gc structures (BrinkmanVan der Linden et al., 2000), and certain viruses use terminal O-acetylated Sias for binding (Cornelissen et al., 1997; de Groot, 2006; Herrler et al., 1985; Regl et al., 1999; Rogers et al., 1986; Schwegmann-Wessels and Herrler, 2006; Suzuki, 2005; Vlasak et al., 1988).

The simplest way of describing changes in the sialome is by quantification of sialylation level under given conditions. This is usually achieved by releasing Sias from the sample either by enzymatic treatment or by acid hydrolysis. Enzymatic treatment yields lower amounts of release, and for some Sia modifications (i.e., 4-O-acetylation) most enzymes do not work at all (Kleineidam et al., 1990). Free Sias can be quantified by thin layer chromatography (TLC), gas-liquid chromatography, mass spectrometry (MS), nuclear magnetic resonance (NMR), or by colorimetric assays (Reuter and Schauer, 1994). A particularly sensitive method is fluorescent derivatization of monosaccharides of the reducing termini of oligosaccharides with 1,2-diamino-4,5-methylenedioxybenzene dihydrochloride (DMB) (Hara et al., 1986). The reducing terminus of polysialic acid can be also be derivatized with DMB by using milder conditions (Inoue et al., 2001). The DMB-Sias are then analyzed using high-pressure liquid chromatography (HPLC). One caveat to studies of released Sias using the methods described above, is that some Sia modifications such as $\mathrm{O}$-acetylation, $\mathrm{O}$-lactylation, and sulfation are sensitive to the acidic conditions employed during Sia release, purification or analysis, and are often inadvertently removed (Lewis and Varki, 2010). In addition, $\mathrm{O}$-acetyl esters at the C-7 position are known to migrate along the Sia side chain to the C-9 a

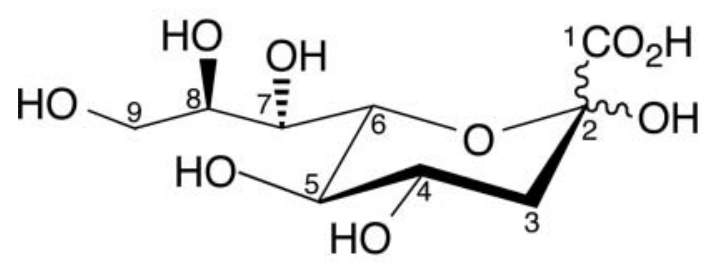

2-Keto-3-deoxynononic acid (Kdn)

C

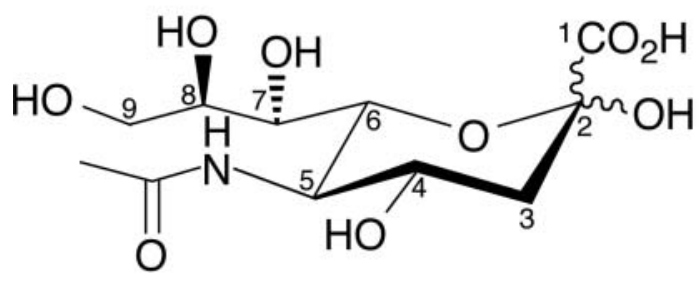

$\mathrm{N}$-Acetylneuraminic acid (Neu5Ac) b

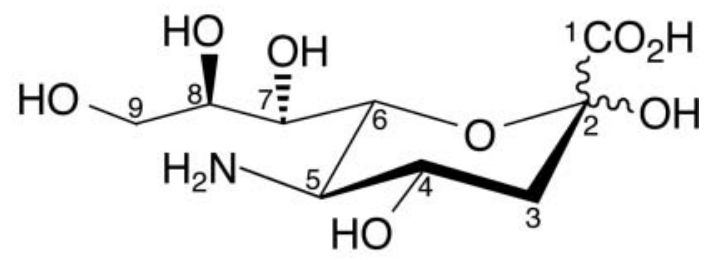

Neuraminic acid (Neu)

d<smiles>O=C(CO)N[C@H]1O[C@H](C(=O)O)[C@H](O)[C@H](O)[C@@H]1O</smiles>

$\mathrm{N}$-Glycolylneuraminic acid (Neu5Gc)

FIG. 1. Sialic acid structure diversity. All known Sias derive from four primary core structures (a) 2-Keto-3-deoxynononic acid (Kdn), (b) neuraminic acid (Neu), (c) N-acetylneuraminic acid (Neu5Ac) and (d) N-glycolylneuraminic acid (Neu5Gc), with potential substitutions at the hydroxyl groups on C-4, C-7, C-8, and C-9 (O-acetyl, O-methyl, O-sulfate, O-lactyl or phosphate groups). Lactones may occur between C-2 and C-7, C-4, or C-8, or a lactam between C-2 and the C-5 amino group of Neu (Angata and Varki, 2002; Varki and Schauer, 2009). 
position, even under physiological conditions (Kamerling et al., 1987; Varki and Diaz, 1984). Finally, other $\alpha$-ketoacids can react with $\mathrm{DMB}$ and give fluorescent peaks eluting in a similar manner on HPLC (Lewis et al., 2009). Thus, interpretation of the sialome at the Sia diversity level should be done with caution, and ideally should include MS confirmation.

\section{Complexity Level 2: Sialic Acid Linkage (the Stems)}

The second layer of complexity of the sialome results from the different $\alpha$-linkages between C-2 and underlying structures (Harduin-Lepers et al., 2005, 2008; Tsuji et al., 1996). The most common linkages are $\alpha 2-3$ or $\alpha 2-6$ to various sugars, or $\alpha 2-8$ to another Sia (Fig. 2). Sia can also form $\alpha 2-9$ linkage to another Sia, and $\alpha 2-4$ linkages occur in the repeating units of some echinodermal glycans (Varki and Schauer, 2009). Each linkage results in a unique spatial presentation of the Sia, which is specifically recognized by GBPs. Binding specificity of influenza virus hemagglutinin, for example, is determined by Sia linkage to the underlying structure: human adapted viruses preferably bind Sias in $\alpha 2-6$ linkage while avian adapted viruses bind Sias in $\alpha 2-3$ linkage (Chandrasekaran et al., 2008; Daniels et al., 1987; Nicholls et al., 2008; Stevens et al., 2006; Suzuki, 2005). NMR spectroscopy is the gold standard for Sia linkage analysis, if enough material is available (Kamerling and Gerwig, 2006). Alternatively, linkage analysis can be done by subjecting sialylated glycans to methylation followed by gas chromatography and mass spectrometry (GC/MS) (Kamerling and Gerwig, 2006; Schauer et al., 1984). Linkage-specific sialidases can also be used to release Sia from a specific linkage, and released Sia molecules can then be quantified. Another approach utilizes GBPs with known specificity for Sia in a certain linkage. Plant lectins such as Sambucus nigra (elderberry bark, SNA) (Shibuya et al., 1987), and Maackia amurensis agglutinin (Knibbs et al., 1991; Wang and Cummings, 1988) are commonly used to detect Sias in $\alpha 2-6$ and $\alpha 2-3$ linkages, respectively. Major caveats for this approach, however, are that the binding specificity of GBPs and lectins is affected by the underlying sugars as well as by glycan density (Dam and Brewer, 2008; Manimala et al., 2007; Taylor and Drickamer, 2009). In addition, the exact specificity of the Maackia amurensis agglutinins can depend on the isoform and source, and crossreactions with sulfated glycans are possible (Bai et al., 2001; BrinkmanVan der Linden et al., 2002).

\section{Complexity Level 3: Underlying Glycan Chain (the Branches)}

Sias are most commonly $\alpha 2-3$ or $\alpha 2-6$ linked to galactose (Gal) or $\alpha 2-6$ linked to $\mathrm{N}$-acetylgalactosamine (GalNAc). However Sia can also be found linked to $\mathrm{N}$-acetylglucosamine (GlcNAc) or to another Sia in $\alpha 2-8$ or $\alpha 2-9$ linkage. These underlying sugars (Fig. 3c) are often part of branched structures comprised of multiple saccharides. The underlying sugar chain can also contribute to the binding specificity of certain GBPs, adding another level of functional complexity to the sialome. For example, selectins preferentially recognize sialylated glycans with an underlying Lewis $\mathrm{X}$ or Lewis A structure (Cummings and Smith, 1992; Hemmerich and Rosen, 2000; Kansas, 1996; Varki, 1994). Recent examples for the effect of underlying sugar chain on binding specificity comes from glycan microarrays, which display a broad range of a Neu5Aco2-3Gal

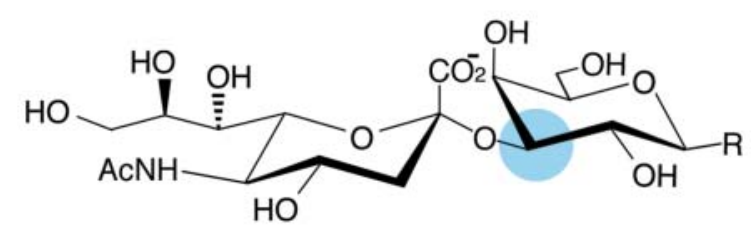

b Neu5Ac $\alpha 2-6 \mathrm{Gal}$

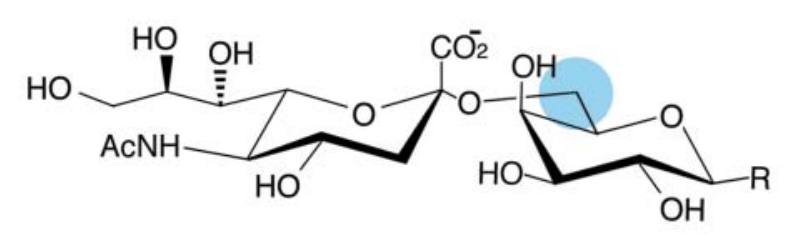

c Neu5Aco2-8Neu5Ac

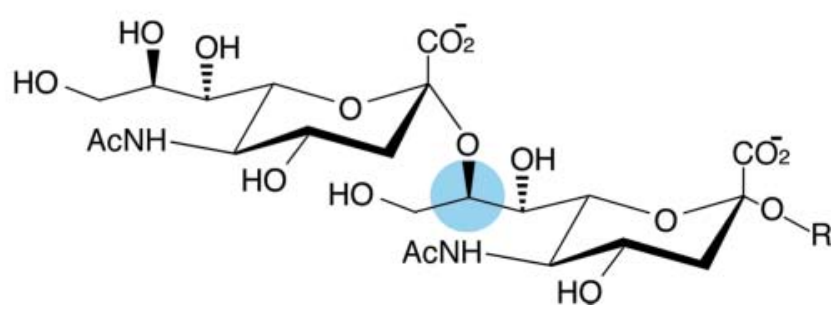

FIG. 2. Sialic acid linkage to underlying sugars. The most common $\alpha$ linkages form between C-2 of Sia and (a) C-3 of Gal, (b) C-6 of Gal or GalNAc, or (c) C-8 of Sia. In the repeating units of some polysaccharides C-2 may form many other kinds of $\alpha$ linkages (Varki and Schauer, 2009). Abbreviations: Galactose (Gal), N-acetylgalactosamine (GalNAc), glucose (Glc).

glycans, either derived from natural sources or chemically synthesized. These microarrays can be interrogated with GBPs or antibodies, or with complex samples such as serum or cell extracts (Horlacher and Seeberger, 2008; Liu et al., 2009). For smaller scale analysis ELISA-like assays can be employed, in which glycan structures are immobilized on an ELISA plate and GBP binding is quantified using a colorimetric assay. Screening of purified hemagglutinin from various influenza strains on a carbohydrate microarray confirmed that the linkage and underlying sugars have a profound effect on binding to a sialylated glycan. For example, human influenza H1(A/Texas/36/1991) hemagglutinin, strongly bound Neu5Ac $2-6 \mathrm{Gal} \beta 1-4 \mathrm{GlcNAc}$ but had weak or no binding if $N$-acetylglucosamine (GlcNAc) was substituted with glucose (Glc) (Stevens et al., 2006). Also, the presence of 6-O-sulfate esters on the Gal or GlcNAc residues of sialyl Lewis structures can greatly facilitate recognition by Siglecs or selectins (Bochner et al., 2005; Hemmerich and Rosen, 2000; Hudson et al., 2009; Kimura et al., 2007). Conversely, sialylated glycan profiles can be analyzed using GBP microarrays (or ELISA-like assays), in which the GBPs (lectins or antibodies) are immobilized on the solid surface and are interrogated with glycan extracts (Pilobello et al., 2007).

Glycan microarrays allow study of the sialome at the third level of complexity, which includes the contribution of the Sia 


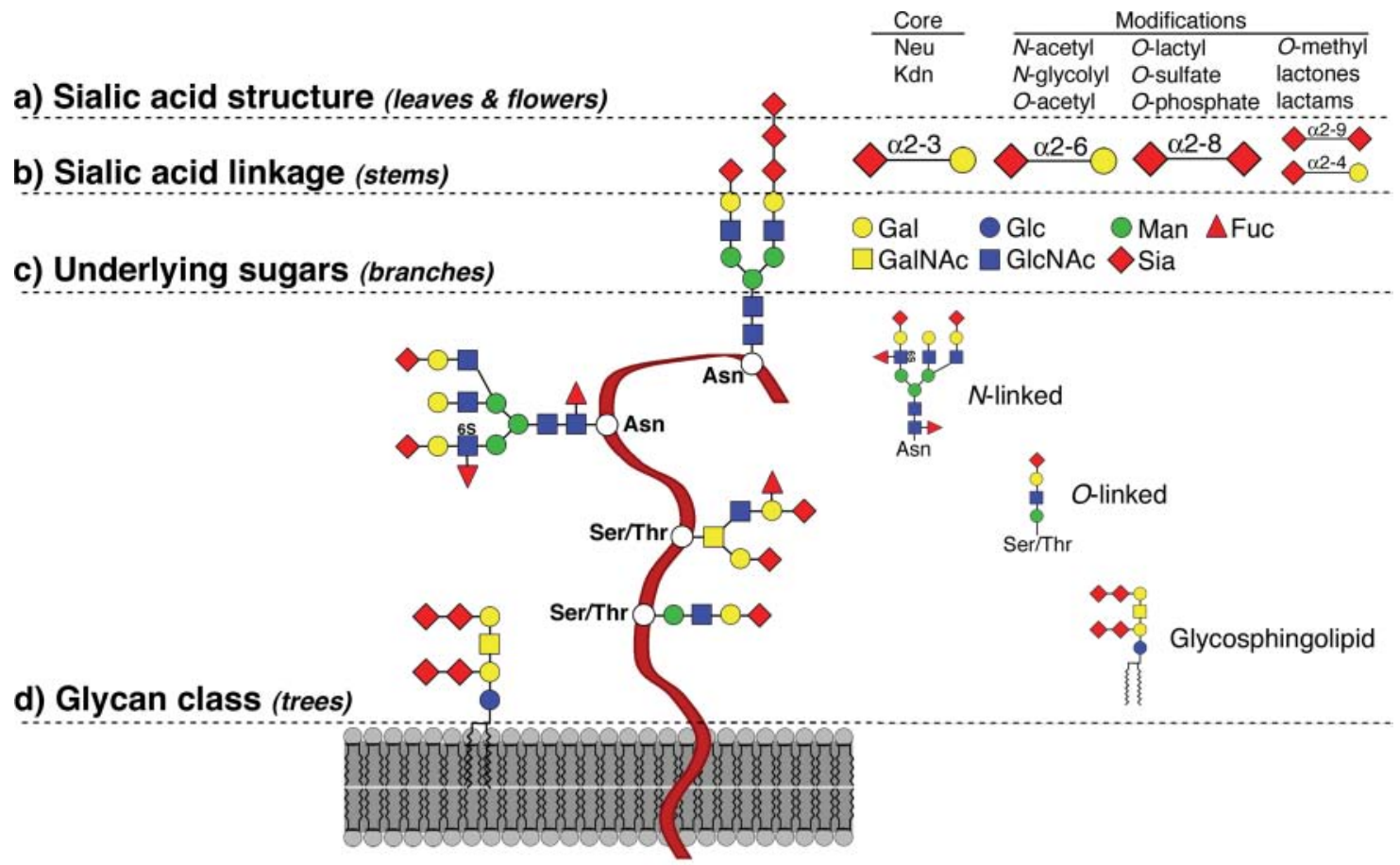

e) Spatial organization (forest)

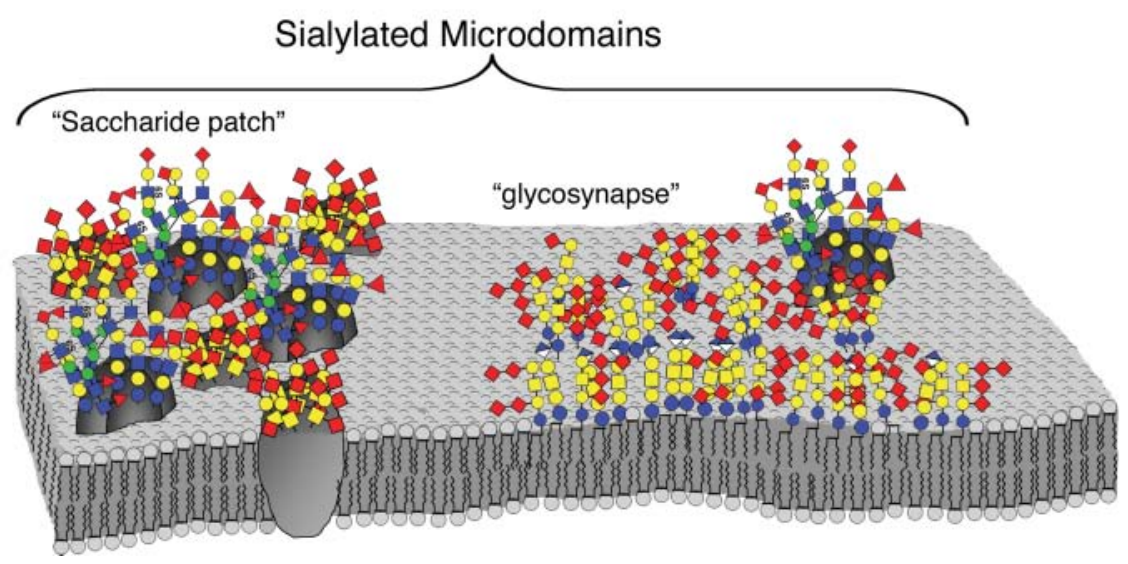

FIG. 3. Hierarchical levels of sialome complexity. The sialome can be analyzed at the following complexity levels: (a) Sia core and core modifications: esterification (with various groups), O-methylation, lactonization, or lactamization yielding $>50$ different structures, (b) Linkage to the underlying sugar (three major and many minor linkages), (c) Identity and arrangement of the underlying sugars that can also be further modified by fucosylation or sulfation, (d) Glycan class ( $N$-linked, $O$-linked or glycosphingolipids), (e) spatial organization of the Sia in sialylated microdomains (including glycosynapses and clustered saccharide patches), and higher levels of the cellular and organismal milieu. Abbreviations: Galactose (Gal), Nacetylgalacosamine (GalNAc), glucose (Glc), N-acetylglucosamine (GlcNAc), mannose (Man), sialic acid (Sia), fucose (Fuc), asparagine (Asn), serine (Ser), threonine (Thr).

core structure and Sia modification, the linkage to the underlying sugar and the underlying sugar structures. Microarray screening allows meaningful high-throughput analysis of biomedically important systems. In addition, because the sialome can change in response to pathological or physiological conditions, such screening may be used to identify unique sialome presentations corresponding to each condition (Pilobello et al., 2007). However arrays can also generate artifacts due to unnaturally large densities of a single sialoglycan. This may cause part of the glycan underlying structure to be inaccessible for binding, resulting both false- positive and false-negative results (Dam and Brewer, 2008; Manimala et al., 2007; Taylor and Drickamer, 2009).

\section{Complexity Level 4: Glycan Class (the Trees)}

Sialylated glycans are typically the terminating units of $\mathrm{N}$-glycans, O-glycans, glycosphingolipids (gangliosides), or sometimes the side chain of glycosylphosphatidylinositol (GPI) anchors (Fuster and Esko, 2005). N-Glycans are covalently attached to an asparagine residue on many secreted and membrane-bound glycoproteins, and carry multiple branches 
on the $\mathrm{Man}_{3} \mathrm{GlNAC}_{2} \mathrm{Asn}$ core structure (Stanley et al., 2009). Sialylated O-glycans are typically GalNAc units covalently attached to serine and threonine residues on glycoproteins often found in mucus secretions, and on cell surface transmembrane glycoproteins with the glycans exposed to the external milieu. Mucins can appear as large gel-forming polymers or soluble smaller oligomers (Brockhausen et al., 2009). Another type of $O$-glycan is $O$-linked mannose, which accounts for a major fraction of all $O$-linked glycans in the brain. Glycosphingolipids are comprised of a glycosylated ceramide lipid moiety, and are primarily found in the outer leaflet of the plasma membrane with the glycans exposed to the external milieu (Schnaar et al., 2009). GPI anchor structures can also sometimes carry Sias on their side chains (Ferguson et al., 2009).

This underlying glycan class can significantly affect Sia presentation, localization and density. For example, P-selectin recognition of PSGL-1 requires a single specific sialyl Lewis $\mathrm{X}$-bearing $\mathrm{O}$-glycan, along with nearby sulfated tyrosine residues (Leppänen et al., 2000). Similarly, the invasive merozoite erythrocyte-binding antigen (EBA)-175 of Plasmodium falciparum (the major cause of malaria mortality), recognizes Neu5Ac $\alpha 2-3 \mathrm{Gal}$ on O-linked tri- and tetra-saccharides on the extracellular domain of glycophorins A (Klotz et al., 1992; Tolia et al., 2005).

High-resolution mass spectrometry methods are often used for glycan class analysis (Haslam et al., 2006; Zaia, 2008). Glycans are released from the sample by specific enzymes or chemical treatment, and then can be subject to MALDI fingerprinting with MS/MS for more details. The use of specific enzymes also allows analysis of $\mathrm{N}$ - and $\mathrm{O}$-glycosylation (Haslam et al., 2006; Zaia, 2008). NMR analysis can also be used for glycan class analysis (De Beer et al., 1994; Petrescu et al., 2006). Malignant transformations are often associated with changes in the glycome, and MS analysis can be used for fast and sensitive screening of serum and urine samples in attempt to identify glycan fingerprinting for early detection of malignant cancer (Haslam et al., 2006). Recent developments in this field include the use of protein chip technology (SELDI) and Fourier-transform ion cyclotron resonance mass spectrometry (FTICR-MS) (Haslam et al., 2006).

Despite the benefit and great contribution of these analytical methods, glycoconjugates have to be extracted from the tissue in order to perform the analysis, which limits understanding of the sialome complexity level. In addition, some of these methods result in destruction of Sia complexity (e.g., loss of $O$-acetylation).

\section{Complexity Level 5: Spatial Organization at the Cell Surface (the Forest Canopy)}

Monovalent glycan ligands typically do not have high affinity interactions with GBPs, and multivalency of the ligands can enhance binding (Brewer et al., 2002; Collins and Paulson, 2004; Dam and Brewer, 2008; Maaheimo et al., 1995; Varki, 1994). However, it was suggested that glycans can also form "clustered saccharide patches" in a variety of different modes (Varki, 1994). Indeed, cell membrane glycans may form dynamic microdomains (Fig. 3e), which mediate specific interaction (Cohen et al., 2009; Hakomori, 2003, Hakomori, 2004). A well-recognized family of sialylated clusters are termed "Glycosynapses," which may be comprised of glycolipids (type 1 glycosynapse), O-linked mucin-type glycoproteins associated with Src family kinases (type 2 glycosynapse) or a complex of gangliosides, integrin receptor and tetraspanin (type 3 glycosynapse) (Todeschini and Hakomori, 2008). Glycosynapses are known to mediate cell signaling and are involved in processes such as cell adhesion, motility and growth (Todeschini and Hakomori, 2008). In another case of "clustered saccharide patches," antibodies may recognize and bind to a complex of two or more sialylated glycans but fail to bind the individual sialoglycans. For example, patients with Guillain Barré or Miller Fisher syndrome produce antibodies specific for ganglioside complexes, including GQ1b/GM1 and GQ1b/GD1a, but which do not bind to the single isolated gangliosides (Kaida et al., 2004; Kanzaki et al., 2008). Sialome recognition at this level of complexity may be analyzed using combinatorial glycoarrays, an array of heterodimeric mixtures of gangliosides compared with single ganglioside (Rinaldi et al., 2009). These microarrays can be interrogated with purified GBPs or antibodies, revealing remarkable examples of such phenomena.

Sialylation can also alter the formation of certain glycan microdomains, such as the galectin-glycoprotein lattice, which form by crosslinking of nonsialylated glycoproteins by multivalent galectins (Brewer et al., 2002; Rabinovich et al., 2007). Galectin-1-glycoprotein lattice formation on $T$ cells induces cell death (Perillo et al., 1995). Galectin-1, however does not bind to $\alpha 2-6$-sialylated structures (Stowell et al., 2008). Thus, sialylation can inhibit Galecin-1-glycoprotein lattice formation and cell death (Rabinovich et al., 2007; Toscano et al., 2007).

Glycans are very dynamic molecules in aqueous solution, and can populate several conformational states under physiological conditions (Chandrasekaran et al., 2008; DeMarco and Woods, 2009). Computer simulation of the conformational dynamics of GM3 ganglioside predicts that membrane anchoring markedly alters the accessibility of carbohydrate residues to solvent and protein binding, without changing the internal structure of the glycan (DeMarco and Woods, 2009). This highlights the importance of directly studying the sialome on the intact cell or tissue. Cells and tissues can be directly probed with specific lectins, recombinant soluble microbial toxins or mammalian receptors, or antibodies. Based on the probe specificity, the spatial distribution of Sia structure, linkage, underlying sugar and glycan class can be identified. Pretreatment with specific glycosidases or chemical modifications of the sample (e.g., periodate treatment) can be used to confirm the binding specificity. Whole-cell lectin microarrays were also developed for high throughput analysis and rapid profiling of glycosylation in a wide range of biological systems (Hsu and Mahal, 2009). These microarrays consist of immobilized lectins, which are interrogated with a complex biological sample, such as virus, bacteria, or tissue extracted glycoproteins, which are fluorescently labeled (Hsu et al., 2006; Hsu and Mahal, 2009).

\section{Sialylated Clusters on Cells Mediate Differential Binding}

At the highest hierarchal level of the sialome, cells with similar Sia content in terms of Sia core structure, modifications, linkages, underlying sugars, and underlying glycan types may still be differentially recognized by GBPs. As an example of such a higher order complexity level, we will 
discuss the sialome of a very simple cell, the human erythrocyte [red blood cell (RBC)]. The erythrocyte membrane presents five major membrane glycoproteins, three of which are heavily sialylated (glycophorins A, B, and C) and two that are not sialylated, but are the major carriers of the $\mathrm{ABH}(\mathrm{O})$ blood group antigens (band 3 and band 4.5) (Anstee, 1990). A, $\mathrm{B}$, and $\mathrm{H}$ antigens are terminal neutral glycan sequences, which determine the ABO blood type polymorphism (Greenwell, 1997; Milland and Sandrin, 2006; Saitou and Yamamoto, 1997; Yamamoto, 2004). Despite the fact that no major variations in Sia content between erythrocytes of $\mathrm{ABO}$ blood groups were reported (Bulai et al., 2003; Cohen et al., 2009), we found that certain Sia binding proteins with the same binding specificity in vitro differentially bind to erythrocytes from the three blood groups (Cohen et al., 2009). Human Siglec-2 (CD22) (Brinkman-Van der Linden et al., 2000; Crocker et al., 2007), which is specific for binding Sia $\alpha 2-$ $6 \mathrm{Gal}$, differentially binds to erythrocytes from each blood type $(\mathrm{A}>\mathrm{O}>\mathrm{B})$. The plant lectin SNA, which has the same binding specificity as Siglec-2 in ELISA assay, also differentially binds to erythrocytes but with binding preference distinct from Siglec-2 (A > B > O) (Cohen et al., 2009). In addition, pfEBA175 , which is specific for binding O-linked Neu5Ac $\alpha 2-3 \mathrm{Gal}$ on glycophorin A (Klotz et al., 1992; Tolia et al., 2005) preferably binds blood type $\mathrm{O}$ erythrocytes (Cohen et al., 2009). In contrast, the pandemic human influenza hemagglutinin (1918South Carolina, also specific for Sia $\alpha 2-6 \mathrm{Gal}$ ) (Stevens et al., 2006), binds to all erythrocytes of all three blood groups to the same extent (Cohen et al., 2009). None of these proteins directly binds A, B, or H antigens. Furthermore, treatment of RBCs with enzymes that selectively convert the $\mathrm{A}$ and $\mathrm{B}$ structures into $\mathrm{O}$ structures also convert the binding of Sia recognizing GBPs in the expected direction. The differential binding is thus attrib- uted to the formation of sialylated "saccharide patches" unique for each blood type (Figs. 3e and 4), which are stabilized by the $\mathrm{ABH}$ antigens (Cohen et al., 2009).

As Sias are involved in many physiological and pathological interactions, other glycans, such as $\mathrm{ABH}$ antigens, can indirectly affect many of these processes by stabilizing unique sialylated clusters. Thus, sialome analysis at this complexity level can explain differential binding specificity to cells with otherwise similar glycan content. For example, microbial interactions have been suggested as a selective force for the persistence of $\mathrm{ABH}(\mathrm{O})$ blood group polymorphisms in humans and other primates. However, direct interactions of microbes with $\mathrm{ABH}$ antigen structures are only known for some strains of Helicobacter pylori (Aspholm-Hurtig et al., 2004) and certain noroviruses (Choi et al., 2008; Huang et al., 2003). Both of these cause nonlethal diseases, which should not have a major impact on reproductive fitness and natural selection. Thus, they cannot explain the evolutionary persistence of the $\mathrm{ABO}$ blood group polymorphisms in the human population, nor their maintenance throughout primate evolution. However, by stabilizing sialylated clusters, $\mathrm{ABH}$ antigens can modulate other critical cellular interactions without being a direct ligand themselves.

\section{Concluding Remarks}

Sialome profiling has to combine the information retrieved from all levels of complexity, and requires constant development of advanced profiling and structural characterization strategies. Because Sia biosynthesis is not template driven and is not encoded directly in the genome, direct labeling of glycans is not trivial. Recently, sialylated glycans labeling on living cells was achieved by mild periodate oxidation
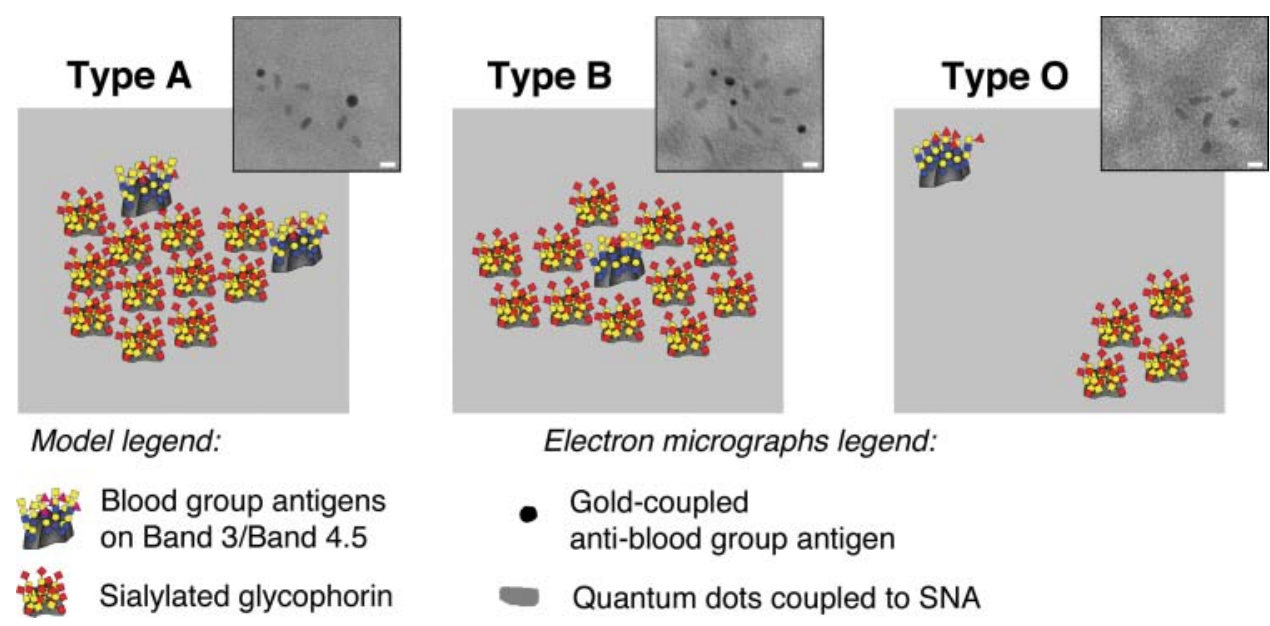

Electron micrographs legend:

- Gold-coupled anti-blood group antigen

- Quantum dots coupled to SNA

FIG. 4. Clustered saccharide patches on cell surfaces. There is no apparent difference in the sialome of human RBCs with blood groups A, B, or O when analyzed at the levels of Sia structure, Sia linkage, underlying sugars, or glycan class. However, at the spatial organizational level, the sialome appears unique for RBCs from each blood type. Sialylated glycans and $\mathrm{ABH}$ blood group antigens seem to be organized in clusters, which are likely stabilized by carbohydrate-carbohydrate interactions with the type A and B terminal saccharides. Left: on blood type A RBCs, the A antigens are apparently localized at the periphery of the cluster, whereas Sias form a continuous cluster that yields a local increase in the Sia concentration. Middle: on blood type B RBCs, the B antigen is apparently localized in the center of the Sia cluster in $\sim 60 \%$ of the clusters. Right: on blood type O RBCs, the Sias clusters are relatively small and may not include the $\mathrm{H}$ antigen. This figure was modified from Cohen et al. (2009). Inserts are electron micrographs of RBCs labeled with anti-blood group antigens followed by gold-conjugated secondary antibody (black), and biotinylated Sambucus nigra agglutinin (SNA) followed by streptavidinconjugated quantum dots 655 (gray). Scale bar represents $10 \mathrm{~nm}$. 
followed by aniline-catalyzed oxime ligation with a fluorescent tag (Zeng et al., 2009). Another approach is based on the promiscuity of glycosylation pathways, which allow processing of bio-orthogonal chemical reporters into natural glycan structures (Prescher and Bertozzi, 2006). The bio-orthogonal reporter is a monosaccharide, which is modified with a functional group (the reporter) that is chemically inert in biological systems. Cells and even whole organism (e.g., zebra fish) can be metabolically labeled with such reporters, which are easily detected with a suitable probe (Jacobs et al., 2000; Prescher and Bertozzi, 2006). Although these and other chemical modification of sialoglycans allow detection and visualization, they also introduce changes to the sugar structure, which may change the sialome organization. Sialylated microdomains, that is, glycosynapses and saccharide patches, are likely stabilized by carbohydrate-carbohydrate interactions (Cohen et al., 2009; Hakomori, 2004), which are sensitive to perturbations. On the other hand, the use of multivalent GBPs might reorganize the sialome. Thus, an "uncertainty principle" applies to all such studies, and most current methods to study the sialome at the highest levels will, by definition, perturb the sialome.

In this review sialome analysis was discussed at five hierarchal complexity levels (Fig. 3), which can be a framework extended to the analysis of other aspects of the glycome as well. Although an abundance of data regarding the sialome was acquired over the years, an up-to-date database containing detailed information has not been constructed. Building such a database is a tedious task that must also reflect the methodology limitations. However, once constructed, it would be of great value (Lewis and Varki, 2010).

\section{Acknowledgments}

We gratefully acknowledge helpful comments from Pascal Gagneux, Sandra Diaz and Oliver Pearce.

\section{Author Disclosure Statement}

The authors declare that no conflicting financial interests exist.

\section{References}

Angata, T., and Varki, A. (2002). Chemical diversity in the sialic acids and related alpha-keto acids: an evolutionary perspective. Chem Rev 102, 439-469.

Anstee, D.J. (1990). The nature and abundance of human red cell surface glycoproteins. J Immunogenet 17, 219-225.

Aspholm-Hurtig, M., Dailide, G., Lahmann, M., Kalia, A., Ilver, D., Roche, N., et al. (2004). Functional adaptation of BabA, the $H$. pylori $\mathrm{ABO}$ blood group antigen binding adhesin. Science 305, 519-522.

Bai, X.M., Brown, J.R., Varki, A., and Esko, J.D. (2001). Enhanced 3-O-sulfation of galactose in Asn-linked glycans and Maackia amurenesis lectin binding in a new Chinese hamster ovary cell line. Glycobiology 11, 621-632.

Bochner, B.S., Alvarez, R.A., Mehta, P., Bovin, N.V., Blixt, O., White, J.R., et al. (2005). Glycan array screening reveals a candidate ligand for siglec-8. J Biol Chem 280, 4307-4312.

Brewer, C.F., Miceli, M.C., and Baum, L.G. (2002). Clusters, bundles, arrays and lattices: novel mechanisms for lectinsaccharide-mediated cellular interactions. Curr Opin Struct Biol 12, 616-623.
Brinkman-Van der Linden, E.C.M., Sjoberg, E.R., Juneja, L.R., Crocker, P.R., Varki, N., and Varki, A. (2000). Loss of Nglycolylneuraminic acid in human evolution-implications for sialic acid recognition by siglecs. J Biol Chem 275, 8633-8640.

Brinkman-Van der Linden, E.C.M., Sonnenburg, J.L., and Varki, A. (2002). Effects of sialic acid substitutions on recognition by Sambucus nigra agglutinin and Maackia amurensis hemagglutinin. Anal Biochem 303, 98-104.

Brockhausen, I., Schachter, H., and Stanley, P. (2009). O-GalNAc Glycans. In Essentials of Glycobiology. A.Varki, R.D. Cummings, J.D. Esko, H.H. Freeze, P. Stanley, C.R. Bertozzi, G.W. Hart, and M.E. Etzler, eds. (Cold Spring Harbor Laboratory Press, Cold Spring Harbor, NY), pp. 115-128.

Bulai, T., Bratosin, D., Pons, A., Montreuil, J., and Zanetta, J.P. (2003). Diversity of the human erythrocyte membrane sialic acids in relation with blood groups. FEBS Lett 534, 185-189.

Chandrasekaran, A., Srinivasan, A., Raman, R., Viswanathan, K., Raguram, S., Tumpey, T.M., et al. (2008). Glycan topology determines human adaptation of avian $\mathrm{H} 5 \mathrm{~N} 1$ virus hemagglutinin. Nat Biotechnol 26, 107-113.

Choi, J.M., Hutson, A.M., Estes, M.K., and Prasad, B.V. (2008). Atomic resolution structural characterization of recognition of histo-blood group antigens by Norwalk virus. Proc Natl Acad Sci USA 105, 9175-9180.

Cohen, M., Hurtado-Ziola, N., and Varki, A. (2009). ABO blood group glycans modulate sialic acid recognition on erythrocytes. Blood 114, 3668-3676.

Collins, B.E., and Paulson, J.C. (2004). Cell surface biology mediated by low affinity multivalent protein-glycan interactions. Curr Opin Chem Biol 8, 617-625.

Cornelissen, L.A.H.M., Wierda, C.M.H., Van, D.M.F.J., Herrewegh, A.A. P.M., Horzinek, M.C., Egberink, H.F., et al. (1997). Hemagglutinin-esterase: A novel structural protein of torovirus. J Virol 71, 5277-5286.

Crocker, P.R., Paulson, J.C., and Varki, A. (2007). Siglecs and their roles in the immune system. Nat Rev Immunol 7, 255266.

Cummings, R.D., and Smith, D.F. (1992). The selectin family of carbohydrate-binding proteins: structure and importance of carbohydrate ligands for cell adhesion. BioEssays 14, 849-856.

Dam, T.K., and Brewer, C.F. (2008). Effects of clustered epitopes in multivalent ligand-receptor interactions. Biochemistry 47, 8470-8476.

Daniels, P.S., Jeffries, S., Yates, P., Schild, G.C., Rogers, G.N., Paulson, J.C., et al. (1987). The receptor-binding and membranefusion properties of influenza virus variants selected using anti-haemagglutinin monoclonal antibodies. EMBO J 6, 14591465.

De Beer, T., Van Zuylen C.W., Hård, K., Boelens, R., Kaptein, R., Kamerling, J.P., et al. (1994). Rapid and simple approach for the NMR resonance assignment of the carbohydrate chains of an intact glycoprotein: application of gradient-enhanced natural abundance $1 \mathrm{H}-13 \mathrm{C}$ HSQC and HSQC-TOCSY to the alpha-subunit of human chorionic gonadotropin. FEBS Lett 348, 1-6.

De Groot, R.J. (2006). Structure, function and evolution of the hemagglutinin-esterase proteins of corona- and toroviruses. Glycoconj J 23, 59-72.

DeMarco, M.L., and Woods, R.J. (2009). Atomic-resolution conformational analysis of the GM3 ganglioside in a lipid bilayer and its implications for ganglioside-protein recognition at membrane surfaces. Glycobiology 19, 344-355.

Ferguson, M.A.J., Kinoshita, A.T., and Hart, G.W. (2009). Glycosylphosphatidylinositol anchors. In Essentials of Glycobiology. 
A.Varki, R.D. Cummings, J.D. Esko, H.H. Freeze, P. Stanley, C.R. Bertozzi, G.W. Hart, and M.E. Etzler, eds. (Cold Spring Harbor Laboratory Press, Cold Spring Harbor, NY), pp. 143162.

Fuster, M.M., and Esko, J.D. (2005). The sweet and sour of cancer: glycans as novel therapeutic targets. Nat Rev Cancer 5, 526-542.

Greenwell, P. (1997). Blood group antigens: molecules seeking a function? Glycoconj J 14, 159-173.

Hakomori, S. (2003). Structure, organization, and function of glycosphingolipids in membrane. Curr Opin Hematol 10, $16-24$.

Hakomori, S. (2004). Carbohydrate-to-carbohydrate interaction, through glycosynapse, as a basis of cell recognition and membrane organization. Glycoconj J 21, 125-137.

Hara, S., Yamaguchi, M., Takemori, Y., Nakamura, M., and Ohkura, Y. (1986). Highly sensitive determination of N-acetyland N-glycolylneuraminic acids in human serum and urine and rat serum by reversed-phase liquid chromatography with fluorescence detection. J Chromatogr 377, 111-119.

Harduin-Lepers, A., Mollicone, R., Delannoy, P., and Oriol, R. (2005). The animal sialyltransferases and sialyltransferaserelated genes: a phylogenetic approach. Glycobiology 15, 805-817.

Harduin-Lepers, A., Petit, D., Mollicone, R., Delannoy, P., Petit, J.M., and Oriol, R. (2008). Evolutionary history of the alpha2,8sialyltransferase (ST8Sia) gene family: tandem duplications in early deuterostomes explain most of the diversity found in the vertebrate ST8Sia genes. BMC Evol Biol 8, 258.

Haslam, S.M., North, S.J., and Dell, A. (2006). Mass spectrometric analysis of $\mathrm{N}$ - and O-glycosylation of tissues and cells. Curr Opin Struct Biol 16, 584-591.

Hemmerich, S., and Rosen, S.D. (2000). Carbohydrate sulfotransferases in lymphocyte homing. Glycobiology 10, 849-856.

Herrler, G., Rott, R., Klenk, H.D., Muller, H.P., Shukla, A.K., and Schauer, R. (1985). The receptor-destroying enzyme of influenza $\mathrm{C}$ virus is neuraminate-O-acetylesterase. EMBO J 4, 1503-1506.

Horlacher, T., and Seeberger, P.H. (2008). Carbohydrate arrays as tools for research and diagnostics. Chem Soc Rev 37, 14141422.

Hsu, K.L., and Mahal, L.K. (2009). Sweet tasting chips: microarray-based analysis of glycans. Curr Opin Chem Biol 13, 427-432.

Hsu, K.L., Pilobello, K.T., and Mahal, L.K. (2006). Analyzing the dynamic bacterial glycome with a lectin microarray approach. Nat Chem Biol 2, 153-157.

Huang, P., Farkas, T., Marionneau, S., Zhong, W., RuvoenClouet, N., Morrow, A.L., et al. (2003). Noroviruses bind to human $\mathrm{ABO}$, Lewis, and secretor histo-blood group antigens: identification of 4 distinct strain-specific patterns. J Infect Dis $188,19-31$.

Hudson, S.A., Bovin, N.V., Schnaar, R.L., Crocker, P.R., and Bochner, B.S. (2009). Eosinophil-selective binding and proapoptotic effect in vitro of a synthetic Siglec-8 ligand, polymeric 6'-sulfated sialyl Lewis x. J Pharmacol Exp Ther 330, 608-612.

Inoue, S., Lin, S.L., Lee, Y.C., and Inoue, Y. (2001). An ultrasensitive chemical method for polysialic acid analysis. Glycobiology 11, 759-767.

Jacobs, C.L., Yarema, K.J., Mahal, L.K., Nauman, D.A., Charters, N.W., and Bertozzi, C.R. (2000). Metabolic labeling of glycoproteins with chemical tags through unnatural sialic acid biosynthesis. Methods Enzymol 327, 260-275.
Kaida, K., Morita, D., Kanzaki, M., Kamakura, K., Motoyoshi, K., Hirakawa, M., et al. (2004). Ganglioside complexes as new target antigens in Guillain-Barre syndrome. Ann Neurol 56, 567-571.

Kamerling, J.P., and Gerwig, G.J. (2006). Structural analysis of naturally occurring sialic acids. Methods Mol Biol 347, 69-91.

Kamerling, J.P., Schauer, R., Shukla, A.K., Stoll, S., Van Halbeek, H., and Vliegenthart, J.F.G. (1987). Migration of O-acetyl groups in N,O-acetylneuraminic acids. Eur J Biochem 162, 601-607.

Kansas, G.S. (1996). Selectins and their ligands: current concepts and controversies. Blood 88, 3259-3287.

Kanzaki, M., Kaida, K., Ueda, M., Morita, D., Hirakawa, M., Motoyoshi, K., et al. (2008). Ganglioside complexes containing GQ1b as targets in Miller Fisher and Guillain-Barre syndromes. J Neurol Neurosurg Psychiatry 79, 1148-1152.

Kelm, S., and Schauer, R. (1997). Sialic acids in molecular and cellular interactions. Int Rev Cytol 175, 137-240.

Kimura, N., Ohmori, K., Miyazaki, K., Izawa, M., Matsuzaki, Y., Yasuda, Y., et al. (2007). Human B-lymphocytes express alpha2-6-sialylated 6-sulfo-N-acetyllactosamine serving as a preferred ligand for CD22/Siglec-2. J Biol Chem 282, 3220032207.

Kleineidam, R.G., Furuhata, K., Ogura, H., and Schauer, R. (1990). 4-Methylumbelliferyl- $\alpha$-glycosides of partially Oacetylated $\mathrm{N}$-acetylneuraminic acids as substrates of bacterial and viral sialidases. Biol Chem Hoppe Seyler 371, 715-719.

Klotz, F.W., Orlandi, P.A., Reuter, G., Cohen, S.J., Haynes, J.D., Schauer, R., et al. (1992). Binding of Plasmodium falciparum 175kilodalton erythrocyte binding antigen and invasion of murine erythrocytes requires $\mathrm{N}$-acetylneuraminic acid but not its O-acetylated form. Mol Biochem Parasitol 51, 49-54.

Knibbs, R.N., Goldstein, I.J., Ratcliffe, R.M., and Shibuya, N. (1991). Characterization of the carbohydrate binding specificity of the leukoagglutinating lectin from Maackia amurensis. Comparison with other sialic acid-specific lectins. J Biol Chem 266, 83-88.

Kornfeld, R., and Kornfeld, S. (1985). Assembly of asparaginelinked oligosaccharides. Annu Rev Biochem 54, 631-664.

Leppänen, A., White, S.P., Helin, J., McEver, R.P., and Cummings, R.D. (2000). Binding of glycosulfopeptides to P-selectin requires stereospecific contributions of individual tyrosine sulfate and sugar residues. J Biol Chem 275, 25967-25958.

Lewis, A.L., Desa, N., Hansen, E.E., Knirel, Y.A., Gordon, J.I., Gagneux, P., et al. (2009). Innovations in host and microbial sialic acid biosynthesis revealed by phylogenomic prediction of nonulosonic acid structure. Proc Natl Acad Sci USA 106, 13552-13557.

Lewis, A.L., and Varki, A. (2010). Evolutionary considerations in studying the sialome: sialic acids and the host-pathogen interface. In An Introduction to Bioinformatics for Glycobiology and Glycomics. C.-W. Von der Lieth, T. Luetteke, and M. Frank, eds. (John Wiley \& Sons, New York).

Liu, Y., Palma, A.S., and Feizi, T. (2009). Carbohydrate microarrays: key developments in glycobiology. Biol Chem 390, 647-656.

Maaheimo, H., Renkonen, R., Turunen, J.P., Penttila, L., and Renkonen, O. (1995). Synthesis of a divalent sialyl Lewis $x$ O-glycan, a potent inhibitor of lymphocyte-endothelium adhesion-evidence that multivalency enhances the saccharide binding to L-selectin. Eur J Biochem 234, 616-625.

Manimala, J.C., Roach, T.A., Li, Z., and Gildersleeve, J.C. (2007). High-throughput carbohydrate microarray profiling of 27 
antibodies demonstrates widespread specificity problems. Glycobiology 17, 17C-23C.

Milland, J., and Sandrin, M.S. (2006). ABO blood group and related antigens, natural antibodies and transplantation. Tissue Antigens 68, 459-466.

Nicholls, J.M., Chan, R.W., Russell, R.J., Air, G.M., and Peiris, J.S. (2008). Evolving complexities of influenza virus and its receptors. Trends Microbiol 16, 149-157.

Perillo, N.L., Pace, K.E., Seilhamer, J.J., and Baum, L.G. (1995). Apoptosis of $\mathrm{T}$ cells mediated by galectin-1. Nature 378, 736-739.

Petrescu, A.J., Wormald, M.R., and Dwek, R.A. (2006). Structural aspects of glycomes with a focus on N-glycosylation and glycoprotein folding. Curr Opin Struct Biol 16, 600607.

Pilobello, K.T., Slawek, D.E., and Mahal, L.K. (2007). A ratiometric lectin microarray approach to analysis of the dynamic mammalian glycome. Proc Natl Acad Sci USA 104, 1153411539.

Prescher, J.A., and Bertozzi, C.R. (2006). Chemical technologies for probing glycans. Cell 126, 851-854.

Rabinovich, G.A., Toscano, M.A., Jackson, S.S., and Vasta, G.R. (2007). Functions of cell surface galectin-glycoprotein lattices. Curr Opin Struct Biol 17, 513-520.

Rademacher, T.W., Parekh, R.B., and Dwek, R.A. (1988). Glycobiology. Annu Rev Biochem 57, 785-838.

Regl, G., Kaser, A., Iwersen, M., Schmid, H., Kohla, G., Strobl, B., et al. (1999). The hemagglutinin-esterase of mouse hepatitis virus strain $\mathrm{S}$ is a sialate-4-O-acetylesterase. J Virol 73, $4721-4727$.

Reuter, G., and Schauer, R. (1994). Determination of sialic acids. Methods Enzymol 230, 168-199.

Rinaldi, S., Brennan, K.M., Goodyear, C.S., O'Leary, C., Schiavo, G., Crocker, P.R., et al. (2009). Analysis of lectin binding to glycolipid complexes using combinatorial glycoarrays. Glycobiology 19, 789-796.

Rogers, G.N., Herrler, G., Paulson, J.C., and Klenk, H.D. (1986). Influenza $C$ virus uses 9-O-acetyl-N-acetylneuraminic acid as a high affinity receptor determinant for attachment to cells. J Biol Chem 261, 5947-5951.

Saitou, N., and Yamamoto, F. (1997). Evolution of primate ABO blood group genes and their homologous genes. Mol Biol Evol 14, 399-411.

Schauer, R. (1982). Chemistry, metabolism, and biological functions of sialic acids. Adv Carbohydr Chem Biochem 40, 131-234.

Schauer, R. (2009). Sialic acids as regulators of molecular and cellular interactions. Curr Opin Struct Biol 19, 507-514.

Schauer, R., Schroder, C., and Shukla, A.K. (1984). New techniques for the investigation of structure and metabolism of sialic acids. Adv Exp Med Biol 174, 75-86.

Schnaar, R.L., Suzuki, A., and Stanley, P. (2009). Glycosphingolipids. In Essentials of Glycobiology. A. Varki, R.D. Cummings, J.D. Esko, H.H. Freeze, P. Stanley, C.R. Bertozzi, G.W. Hart, and M.E. Etzler, eds. (Cold Spring Harbor Laboratory Press, Cold Spring Harbor, NY), pp. 129-142.

Schwegmann-Wessels, C., and Herrler, G. (2006). Sialic acids as receptor determinants for coronaviruses. Glycoconj J 23, $51-58$.

Shibuya, N., Goldstein, I.J., Broekaert, W.F., Nsimba-Lubaki, M., Peeters, B., and Peumans, W.J. (1987). The elderberry (Sambucus nigra L.) bark lectin recognizes the Neu5Ac(alpha 2-6)Gal/GalNAc sequence. J Biol Chem 262, 1596-1601.
Stanley, P., Schachter, H., and Taniguchi, N. (2009). N-Glycans. In Essentials of Glycobiology. A. Varki, R.D. Cummings, J.D. Esko, H.H. Freeze, P. Stanley, C.R. Bertozzi, G.W. Hart, and M.E. Etzler, eds. (Cold Spring Harbor Laboratory Press, Cold Spring Harbor, NY), pp. 101-114.

Stevens, J., Blixt, O., Glaser, L., Taubenberger, J.K., Palese, P., Paulson, J.C., et al. (2006). Glycan microarray analysis of the hemagglutinins from modern and pandemic influenza viruses reveals different receptor specificities. J Mol Biol 355, 11431155.

Stowell, S.R., Arthur, C.M., Mehta, P., Slanina, K.A., Blixt, O., Leffler, H., et al. (2008). Galectin-1, -2 , and -3 exhibit differential recognition of sialylated glycans and blood group antigens. J Biol Chem 283, 10109-10123.

Suzuki, Y. (2005). Sialobiology of influenza: molecular mechanism of host range variation of influenza viruses. Biol Pharm Bull 28, 399-408.

Taylor, M.E., and Drickamer, K. (2009). Structural insights into what glycan arrays tell us about how glycan-binding proteins interact with their ligands. Glycobiology 19, 11551162.

Todeschini, A.R., and Hakomori, S.I. (2008). Functional role of glycosphingolipids and gangliosides in control of cell adhesion, motility, and growth, through glycosynaptic microdomains. Biochim Biophys Acta 1780, 421-433.

Tolia, N.H., Enemark, E.J., Sim, B.K., and Joshua-Tor, L. (2005). Structural basis for the EBA-175 erythrocyte invasion pathway of the malaria parasite Plasmodium falciparum. Cell 122, 183-193.

Toscano, M.A., Bianco, G.A., Ilarregui, J.M., Croci, D.O., Correale, J., Hernandez, J.D., et al. (2007). Differential glycosylation of TH1, TH2 and TH-17 effector cells selectively regulates susceptibility to cell death. Nat Immunol 8, 825834.

Troy, F.A. (1992). Polysialylation: from bacteria to brains. Glycobiology 2, 5-23.

Tsuji, S., Datta, A.K., and Paulson, J.C. (1996). Systematic nomenclature for sialyltransferases. Glycobiology 6, v-vii.

Varki, A. (1992). Diversity in the sialic acids. Glycobiology 2, 25-40.

Varki, A. (1994). Selectin ligands. Proc Natl Acad Sci USA 91, 7390-7397.

Varki, A. (2007). Glycan-based interactions involving vertebrate sialic-acid-recognizing proteins. Nature 446, 1023-1029.

Varki, A. (2008). Sialic acids in human health and disease. Trends Mol Med 14, 351-360.

Varki, A., and Angata, T. (2006). Siglecs-the major subfamily of I-type lectins. Glycobiology 16, 1R-27R.

Varki, A., and Diaz, S. (1984). The release and purification of sialic acids from glycoconjugates: methods to minimize the loss and migration of O-acetyl groups. Anal Biochem 137, 236-247.

Varki, A., and Schauer, R. (2009). Sialic acids. In Essentials of Glycobiology. A. Varki, R.D. Cummings, J.D. Esko, H.H. Freeze, P. Stanley, C.R. Bertozzi, G.W. Hart, and M.E. Etzler, eds. (Cold Spring Harbor Laboratory Press, Cold Spring Harbor, NY), pp.. 199-218.

Varki, A., and Sharon, N. (2009). Historical background and overview. In Essentials of Glycobiology. A. Varki, R.D. Cummings, J.D. Esko, H.H. Freeze, P. Stanley, C.R. Bertozzi, G.W. Hart, and M.E. Etzler, eds. (Cold Spring Harbor Laboratory Press, Cold Spring Harbor, NY), pp. 1-22.

Vlasak, R., Luytjes, W., Spaan, W., and Palese, P. (1988). Human and bovine coronaviruses recognize sialic acid-containing 
receptors similar to those of influenza $C$ viruses. Proc Natl Acad Sci USA 85, 4526-4529.

Wang, W.C., and Cummings, R. D. (1988). The immobilized leukoagglutinin from the seeds of Maackia amurensis binds with high affinity to complex-type Asn-linked oligosaccharides containing terminal sialic acid-linked alpha-2,3 to penultimate galactose residues. J Biol Chem 263, 45764585.

Yamamoto, F. (2004). Review: ABO blood group system-ABH oligosaccharide antigens, anti-A and anti- $\mathrm{B}, \mathrm{A}$ and $\mathrm{B}$ glycosyltransferases, and ABO genes. Immunohematol 20, 3-22.

Zaia, J. (2008). Mass spectrometry and the emerging field of glycomics. Chem Biol 15, 881-892.

Zeng, Y., Ramya, T.N., Dirksen, A., Dawson, P.E., and Paulson, J.C. (2009). High-efficiency labeling of sialylated glycoproteins on living cells. Nat Methods 6, 207-209.
Address correspondence to: Miriam Cohen

University of California, San Diego 9500 Gilman Drive

La Jolla, CA 92093-0687

E-mail: micohen@ucsd.edu

or

Ajit Varki

University of California, San Diego 9500 Gilman Drive La Jolla, CA 92093-0687

E-mail: a1varki@ucsd.edu 